\section{Transtornos psiquiátricos menores em cuidadores familiares de usuários de Centros de Atenção Psicossocial do Sul do Brasil}

\author{
Minor psychiatric disorders in family caregivers of \\ users of Psychosocial Care Centers in \\ southern Brazil
}

\author{
1 Faculdade de Enfermagem \\ e Obstetrícia, Universidade \\ Federal de Pelotas, Pelotas, \\ Brasil. \\ 2 Centro de Pesquisas \\ Epidemiológicas, \\ Universidade Federal de \\ Pelotas, Pelotas, Brasil. \\ Correspondência \\ L. C. M. Quadros \\ Faculdade de Enfermagem \\ e Obstetrícia, Universidade \\ Federal de Pelotas. \\ Rua Barão da Conceição 301, \\ Pelotas, RS \\ 96015-310, Brasil. \\ lenicemuniz@hotmail.com
}

\begin{abstract}
A cross-sectional study was conducted with 936 family caregivers of users of Psychosocial Care Centers (CAPS) with the aim of describing the prevalence of minor psychiatric disorders and the association with socio-demographic variables, self-reported overload, and stressful events. Minor psychiatric disorder was identified with the Self-Reporting Questionnaire (SRQ-20), using as case definition a cutoff of eight or more positive answers for women and six or more for men. Bivariate and multivariate analyses were conducted by Poisson regression. Prevalence of $\mathrm{mi}$ nor psychiatric disorders was 49\% (95\%CI: 46\%52\%). In the adjusted analysis, higher prevalence was observed in families with less schooling, health problems, more stressful life events, retirees, and single caregivers who reported overload. The results indicate a high prevalence of minor psychiatric disorders, besides identifying some associations. Such information provides important support for planning mental health services and primary care.
\end{abstract}

Caregivers; Mental Health Services; Mental Health
Lenice de Castro Muniz de Quadros 1

Denise Petrucci Gigante 2

Luciane Prado Kantorski 1

Vanda Maria da Rosa Jardim 1

\section{Introdução}

A atual Política de Saúde Mental no Brasil apoiase na Lei $n^{\circ}$. 10.216/02 e busca a consolidação de um modelo de atenção à saúde mental aberto, de base comunitária, com uma rede de serviços e equipamentos variados, como os Centros de Atenção Psicossocial (CAPS), os Serviços Residenciais Terapêuticos (SRT), os Centros de Convivência e Cultura, os leitos em Hospitais Gerais e em CAPS III (Ministério da Saúde. http://portal.sau de.gov.br/portal/saúde/área.cfm?id_area=925, acessado em 02/Dez/2009). Com a aprovação da Portaria $n^{\circ}$. 336 do Ministério da Saúde, de fevereiro de 2002, os CAPS foram regulamentados como serviços substitutivos ao modelo manicomial centrado no hospital psiquiátrico, significando uma grande vitória do movimento da reforma psiquiátrica 1 .

Os CAPS são unidades locais/regionalizadas, com uma população adstrita, definida pelo nível local, oferecendo atendimento diário aos portadores de sofrimento psíquico. Essas unidades permitem que os usuários permaneçam junto às suas famílias e comunidade, estimulando sua integração social e familiar, apoiando suas iniciativas de busca da autonomia e oferecendo atendimento médico e psicológico ${ }^{2}$. Assim, a inclusão da família do usuário dos serviços de saúde mental no tratamento faz-se necessária para proporcionar suporte ao usuário bem como para receber cuidados específicos 2,3 . 
Dos fatores que devem ser observados ao prestar o cuidado aos familiares, destaca-se a sobrecarga na prestação de cuidados ao usuário, que pode se traduzir através da prevalência de transtornos psiquiátricos menores 4,5,6. Enquanto esses problemas atingem de $25 \%$ a $38 \%$ de adultos avaliados através de estudos de base populacional realizados no Brasil 7,8 , as prevalências foram mais elevadas (de $34 \%$ a $41 \%$ ) quando em estudos conduzidos com familiares cuidadores 9,10 .

Os principais fatores associados à positividade do Self-Reporting Questionnaire (SRQ-20) têm sido relacionados a: ser do sexo feminino, ter baixa escolaridade, baixa renda, falta de apoio social e situação ocupacional desfavorável 8,11,12,13,14.

Diante do exposto, o presente artigo teve por objetivo descrever a prevalência de transtornos psiquiátricos menores e sua associação com variáveis sociodemográficas, sobrecarga autorreferida e eventos estressores em familiares cuidadores de usuários de CAPS no Sul do Brasil.

\section{Métodos}

Estudo transversal realizado a partir de dados previamente coletados na pesquisa de avaliação dos CAPS da Região Sul (Rio Grande do Sul, Santa Catarina, Paraná) do Brasil. O projeto CAPSUL foi desenvolvido no período de janeiro de 2006 a fevereiro de 2008, incluindo dois subestudos (quantitativo e qualitativo). Para este trabalho utilizaram-se os dados obtidos através do subestudo quantitativo. Através de sorteio aleatório foram escolhidos 30 municípios da região nos quais a coleta de dados foi realizada.

Respeitando o número total de CAPS por estado, a escolha dos municípios foi feita proporcionalmente, observando-se o tipo de serviço, ou seja, a classificação em: CAPS I e CAPS II, de acordo com o número de habitantes do município. Dos 30 municípios selecionados, três serviços eram no Paraná, nove em Santa Catarina e 18 no Rio Grande do Sul.

Nos 30 CAPS incluídos neste estudo foram entrevistados 936 familiares dos usuários desses serviços. Essa amostra permitiu estimar uma prevalência de transtornos psiquiátricos menores de $40 \%$ com erro em torno de três pontos percentuais, para um nível de 95\% de confiança. Para estudar associações com um nível de $95 \%$ de confiança, poder de $80 \%$, razão exposto/não exposto de $1 / 4$, prevalência da doença em não expostos de $20 \%$, essa amostra é suficiente para encontrar um risco relativo de até 1,5.

A coleta de dados foi realizada por $14 \mathrm{du}$ plas de entrevistadores e ocorreu no período de maio a junho de 2006. Treinamento e capacitação foram conduzidos previamente ao trabalho de campo. Estudo piloto realizado em CAPS de Pelotas, não sorteado para constituir a amostra, foi supervisionado com o objetivo de adequar o instrumento e avaliar os entrevistadores em condições reais de coleta de dados.

O controle de qualidade durante o trabalho de campo foi realizado pela checagem ao final de cada entrevista, revisão dos supervisores ao receber o questionário e na correção da codificação. Na entrada dos dados, o controle de qualidade foi através de dupla digitação permitindo a verificação, comparação e correção de possíveis erros.

No instrumento aplicado aos familiares, com questões abertas e fechadas, foi incluído o SRQ20. Esse instrumento foi projetado por Harding et al. 15 e proposto pela Organização Mundial da Saúde para a detecção de transtornos psiquiátricos menores na população. É constituído por vinte questões com respostas do tipo sim ou não para cada uma delas. Tradução e validação para a língua portuguesa foram conduzidas por Mari \& Williams 16 , apresentando sensibilidade de $85 \%$ e especificidade de $80 \%$.

Para definir a prevalência de transtorno psiquiátrico menor utilizou-se o número de respostas positivas em cada uma das questões do SRQ. Assim, as mulheres com oito ou mais respostas positivas nessa escala foram consideradas como possíveis casos de transtorno psiquiátricos menores. Para os homens, esse ponto de corte foi de seis ou mais respostas positivas, conforme sugerido no estudo de validação do instrumento no Brasil 17.

Neste estudo, além dos 20 itens do SRQ-20 foram utilizadas outras informações do instrumento. Assim, foram incluídas variáveis sociodemográficas, tais como sexo (variável dicotômica), idade (menos de 40 anos, 40-49 anos, 50-59 anos, 60 anos ou mais), escolaridade (0-4 anos, 5-8 anos, 9-11 anos, 12 anos ou mais) renda familiar (dividido em quartil, sendo o primeiro considerado o mais baixo e o quarto o mais elevado nível de renda) situação conjugal (com companheiro, sem companheiro) trabalho remunerado (sim/não) vinculo com o usuário [pai/mãe, irmão, cônjuge, filho(a), outros], eventos estressores como morte do companheiro, divórcio ou separação, prisão ou problemas com a justiça, morte de familiar/ amigo intimo, acidentes ou doenças pessoais, acidentes ou doenças na família e perda do emprego, que estavam disponíveis no questionário com respostas do tipo sim ou não e, de acordo com a revisão da literatura. Foram categorizados por número de eventos referidos (nenhum, um, dois e três eventos ou mais), problemas de saúde (sim/não), aposentadoria (sim/não), participa- 
ção em atividades no serviço (sim/não), único cuidador ( $\operatorname{sim} /$ não) e sobrecarga autorreferida (sim/não).

A análise deste estudo foi realizada com o pacote estatístico Stata 11 (Stata Corp., College Station, Estados Unidos), incluindo análises univariada, bivariada e multivariável. Procedeu-se à comparação entre proporções, utilizando-se o teste do qui-quadrado e na presença de variáveis independentes ordinais, foi testada a tendência linear. Regressões de Poisson bruta e ajustada foram conduzidas, sendo esta última de acordo com modelo hierarquizado (Figura 1). Assim, as variáveis do mesmo nível foram ajustadas entre si e, a partir do segundo nível foram mantidas na análise ajustada aquelas que se mantiveram associadas com o desfecho ( $\mathrm{p}<0,2)$, além daquelas do mesmo nível. Considerando uma possível colinearidade entre renda familiar e escolaridade, foram realizadas duas análises ajustadas no primeiro nível. Para inclusão das variáveis no modelo ajustado utilizou-se o valor de p de 0,2, mas o nível de significância foi de $5 \%$ em todos os testes utilizados.

Figura 1

Modelo hierarquizado.

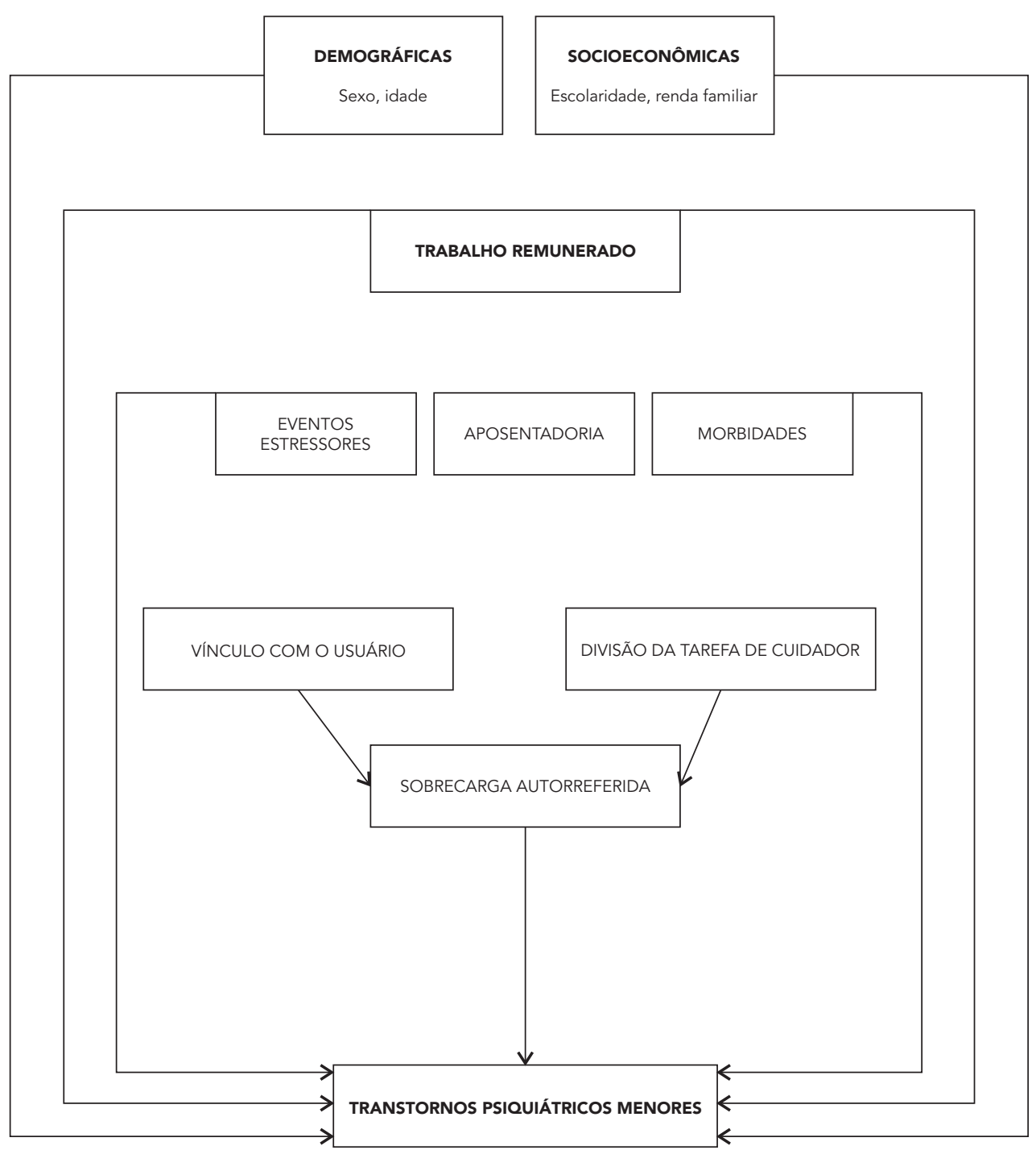


O projeto CAPSUL foi aprovado pelo Comitê de Ética da Faculdade de Medicina da Universidade Federal de Pelotas, sob o ofício de número 074/95. Todos os participantes assinaram o Consentimento Livre e Esclarecido.

\section{Resultados}

Dos 936 familiares entrevistados, 99\%, ou seja, 925 indivíduos responderam todas as questões do SRQ-20. Para esses, a prevalência total de transtornos psiquiátricos menores foi de $49 \%$ (IC95\%: 46\%-52\%).

Os familiares entrevistados pertenciam na sua maioria ao Estado do Rio Grande do Sul, já que é o estado que concentra o maior número de CAPS. Os estados de Santa Catarina e Paraná tiveram respectivamente $22 \%$ e $11 \%$ do total de familiares entrevistados.

A Tabela 1 apresenta a distribuição da amostra por variáveis demográficas e socioeconômicas e a associação destas com os transtornos psiquiá- tricos menores. A maior parcela da amostra foi composta por mulheres, acima dos 50 anos, com baixa escolaridade, que vive com companheiro e não tem trabalho remunerado. Ao dividir a renda familiar por quartil, constatou-se que $25 \%$ da amostra recebeu no ultimo mês até 1,4 salário mínimo. O quartil superior apresentou renda familiar a partir de 3,2 salários mínimos. Chama atenção que o segundo quartil incluiu um pouco mais de 25\%, uma vez que 115 (13\%) dos familiares tiveram renda de dois salários.

Ao analisar as prevalências de transtornos psiquiátricos menores em relação a essas variáveis, observa-se que não houve diferença em homens e mulheres ou se viviam ou não com companheiro. Discretos aumentos nas prevalências de transtornos psiquiátricos menores foram observados com o aumento da idade dos familiares, no entanto a diferença entre as categorias de idade não foi significativa $(\mathrm{p}=0,06)$.

A distribuição da amostra em relação aos eventos da vida, vínculo com usuário e sobrecarga autorreferida é apresentada na Tabela 2.

Tabela 1

Distribuição da amostra por variáveis demográficas e socioeconômicas e transtornos psiquiátricos menores.

\begin{tabular}{|c|c|c|c|c|}
\hline Variável & $\mathbf{n}$ & $\%$ & SRQ positivo (\%) & Valor de $p$ \\
\hline Sexo & & & & 0,25 \\
\hline Masculino & 265 & 28,3 & $120(46,2)$ & \\
\hline Feminino & 671 & 71,7 & $335(50,4)$ & \\
\hline Idade (aos) & & & & $0,06 / 0,01$ * \\
\hline$<40$ & 240 & 25,7 & $101(42,4)$ & \\
\hline $40-49$ & 200 & 21,4 & $95(47,7)$ & \\
\hline $50-59$ & 255 & 27,2 & $134(53,4)$ & \\
\hline 60 ou mais & 240 & 25,7 & $124(52,5)$ & \\
\hline Escolaridade (anos) & & & & $<0,001 /<0,001 *$ \\
\hline $0-4$ & 392 & 43,2 & $231(60,2)$ & \\
\hline $5-8$ & 279 & 30,8 & $120(43,3)$ & \\
\hline $9-11$ & 171 & 18,8 & $68(40,0)$ & \\
\hline 12 ou mais & 65 & 7,2 & $22(33,8)$ & \\
\hline Renda familiar & & & & $0,004 / 0,001$ * \\
\hline 1ㅇ quartil (menor renda) & 216 & 25,1 & $114(53,5)$ & \\
\hline 2o quartil & 243 & 28,2 & $132(55,2)$ & \\
\hline 3ㅇ quartil & 194 & 22,5 & $93(48,4)$ & \\
\hline 4ㅇ quartil & 209 & 24,2 & $81(39,1)$ & \\
\hline Situação conjugal & & & & 0,64 \\
\hline Com companheiro & 559 & 59,7 & $269(48,6)$ & \\
\hline Sem companheiro & 377 & 40,3 & $186(50,1)$ & \\
\hline Trabalho remunerado & & & & 0,004 \\
\hline Sim & 332 & 35,5 & $140(42,8)$ & \\
\hline Não & 603 & 64,5 & $314(52,6)$ & \\
\hline
\end{tabular}

* Teste de tendência linear. 
Distribuições da amostra por variáveis referentes a eventos estressores, vínculo com o usuário, sobrecarga e transtornos psiquiátricos menores.

\begin{tabular}{|c|c|c|c|c|}
\hline Variável & $\mathrm{n}$ & $\%$ & SRO positivo (\%) & Valor de $p$ \\
\hline Eventos estressores & & & & $<0,001$ \\
\hline Nenhum & 249 & 26,7 & $83(33,9)$ & \\
\hline 1 & 303 & 32,5 & $135(45,0)$ & \\
\hline 2 & 227 & 24,4 & $132(58,4)$ & \\
\hline 3 ou mais & 153 & 16,4 & $105(68,6)$ & \\
\hline Problemas de saúde & & & & $<0,001$ \\
\hline Não & 421 & 45,0 & $138(43,9)$ & \\
\hline Sim & 515 & 55,0 & $314(61,7)$ & \\
\hline Aposentadoria & & & & 0,002 \\
\hline Não & 903 & 96,8 & $432(48,3)$ & \\
\hline Sim & 30 & 3,2 & $23(76,7)$ & \\
\hline $\begin{array}{l}\text { Participação em atividade no } \\
\text { serviço }\end{array}$ & & & & 0,90 \\
\hline Sim & 349 & 37,3 & $169(48,9)$ & \\
\hline Não & 586 & 62,7 & $286(49,4)$ & \\
\hline Vínculo com o usuário & & & & 0,03 \\
\hline Pai/Mãe & 392 & 41,9 & $210(54,4)$ & \\
\hline Irmão & 141 & 15,1 & $60(42,9)$ & \\
\hline Cônjuge & 184 & 19,6 & $92(50,5)$ & \\
\hline Filho(a) & 143 & 15,3 & $64(45,1)$ & \\
\hline Outros & 76 & 8,1 & $29(38,7)$ & \\
\hline \multicolumn{5}{|l|}{ Único cuidador } \\
\hline Não & 324 & 34,7 & $138(43,9)$ & 0,006 \\
\hline Sim & 611 & 65,3 & $317(52,6)$ & \\
\hline Se sente sobrecarregado & & & & $<0,001$ \\
\hline Não & 412 & 44,2 & $148(36,3)$ & \\
\hline Sim & 521 & 55,8 & $307(59.5)$ & \\
\hline
\end{tabular}

Observa-se que a maioria referiu algum evento estressor, problema de saúde, não participar de atividades no CAPS, ser parente do usuário em primeiro grau, ser único cuidador e sentir-se sobrecarregado. As prevalências de transtorno psiquiátrico menor foram diferentes para todas essas características incluídas na Tabela 2, exceto para participação em atividades no serviço.

Os resultados da análise de regressão (Tabela 3) mostram que a idade e a renda familiar perdem a significância estatística ao serem ajustadas entre si, sendo mantida no modelo apenas a escolaridade destas variáveis incluídas no primeiro nível de análise. Das variáveis incluídas no próximo nível de análise, o trabalho remunerado perdeu a significância estatística ao ser ajustado para escolaridade. Quanto às variáveis do terceiro e do quarto nível no modelo, eventos estressores, aposentadoria, vínculo com usuário e sobrecarga autorreferida, observa-se que apenas o vínculo com o usuário deixou de ter uma associação significativa quando ajustado para as variáveis do mesmo ou de níveis hierarquicamente superiores (Tabela 4).

\section{Discussão}

O presente estudo mostra a prevalência de transtornos psiquiátricos menores, segundo variáveis sociodemográficas, sobrecarga autorreferida e eventos estressores em familiares cuidadores de usuários de CAPS, através de dados coletados na pesquisa CAPSUL.

A prevalência de transtornos psiquiátricos menores (49\%) foi superior àquelas encontradas por estudos de base populacional realizados no Brasil 8,14, bem como por estudos realizados com 
Razão de prevalência (RP) bruta e ajustada de transtornos psiquiátricos menores, associação com variáveis demográficas e socioeconômicas.

\begin{tabular}{|c|c|c|c|c|}
\hline Variável & RP bruta (IC95\%) & Valor de $p$ & RP ajustada * (IC95\%) & Valor de p \\
\hline Sexo & & 0,25 & & 0,23 \\
\hline Masculino & 1,00 & & 1,00 & \\
\hline Feminino & $1,10(0,93-1,27)$ & & $1,10(0,94-1,29)$ & \\
\hline Idade (anos) & & 0,01 & & $0,61 / 0,52$ ** \\
\hline$<40$ & 1,00 & & 1,00 & \\
\hline $40-49$ & $1,12(0,91-1,38)$ & & $1,02(0,81-1,28)$ & \\
\hline $50-59$ & $1,25(1,04-1,51)$ & & $1,13(0,91-1,40)$ & \\
\hline 60 ou mais & $1,23(1,02-1,49)$ & & $1,04(0,83-1,31)$ & \\
\hline Escolaridade (anos) & & $<0,001$ & & $<0,001 /<0,001$ ** \\
\hline 12 ou mais & 1,00 & & 1,00 & \\
\hline $9-11$ & $1,18(0,80-1,73)$ & & $1,32(0,84-2,06)$ & \\
\hline $5-8$ & $1,27(0,88-1,84)$ & & $1,35(0,88-2,08)$ & \\
\hline $0-4$ & $1,77(1,25-2,52)$ & & $1,86(1,22-2,83)$ & \\
\hline Renda familiar & & 0,007 & & $0,44 / 0,27 * *$ \\
\hline 4으 quartil & 1,00 & & 1,00 & \\
\hline 3o quartil & $1,23(0,99-1,55)$ & & $1,15(0,92-1,44)$ & \\
\hline 2o quartil & $1,41(1,15-1,73)$ & & $1,21(0,98-1,50)$ & \\
\hline 1ㅇ quartil (menor renda) & $1,37(1,11-1,69)$ & & $1,15(0,92-1,44)$ & \\
\hline Trabalho remunerado & & 0,006 & & 0,15 \\
\hline Sim & 1,00 & & 1,00 & \\
\hline Não & $1,22(1,06-1,42)$ & & $1,12(0,96-1,30)$ & \\
\hline
\end{tabular}

IC95\%: intervalo de 95\% de confiança.

* Conforme modelo hierárquico;

** Teste de tendência linear.

cuidadores que utilizaram o mesmo critério de classificação através do SRQ-20 9,10. Cabe destacar que a atenção ao usuário de saúde mental pode gerar maior sobrecarga ao familiar 18, o que pode levar a maior frequência de problemas relacionados à saúde mental.

Quanto ao perfil dos familiares, observase que os resultados encontrados neste estudo mostram semelhanças com aqueles também conduzidos com cuidadores 9,10. Sendo assim é possível constatar o predomínio de mulheres e com vinculo familiar próximo ao usuário.

Enquanto a renda familiar, o trabalho remunerado e a idade, apresentaram somente associação bruta com a positividade no SRQ-20, escolaridade permanece fortemente associada na análise ajustada. Essa forte relação entre escolaridade e transtorno psiquiátrico menor também tem sido observada em outros estudos 13,14.

Embora a maioria dos estudos que foram conduzidos tanto na população em geral como com os cuidadores tem mostrado que os transtornos psiquiátricos menores são mais frequentes em mulheres 12,14,17, essa associação não foi encontrada no presente estudo. Entretanto, cabe destacar que o poder deste estudo para encontrar diferença nessa associação foi em torno de $20 \%$.

Enquanto a ausência de emprego tem sido citada 7 como um dos fatores associados à positividade no SRQ-20, no presente estudo essa associação desaparece quando ajustada para variáveis socioeconômicas e demográficas. Outras funções da inserção laboral, além da falta de remuneração, relacionam ausência de emprego a transtornos psiquiátricos menores. Entre essas, a estruturação temporal do cotidiano e contatos com pessoas fora da família, que transcendem o nível individual, o status e a identidade poderiam funcionar como eixos para organizar a vida do cotidiano19.

Em relação às associações dos transtornos psiquiátricos menores com problemas crônicos de saúde, eventos estressores, e aposentadoria, isso também tem sido observado em outros estudos 7,12,20,21,22.

A associação encontrada no presente estudo entre ser único cuidador e os transtornos psiqui- 
Razão de prevalência (RP) bruta e ajustada de transtornos psiquiátricos menores, associação com variáveis referentes a eventos de vida, vínculo com o usuário e sobrecarga.

\begin{tabular}{|c|c|c|c|c|}
\hline Variável & RP bruta (IC95\%) & Valor de $p$ & RP ajustada * (IC95\%) & Valor de $p$ \\
\hline Eventos estressores & & $<0,001$ & & $<0,001 /<0,001$ ** \\
\hline Nenhum & 1,00 & & 1,00 & \\
\hline 1 & $1,33(1,07-1,65)$ & & $1,33(1,08-1,65)$ & \\
\hline 2 & $1,72(1,40-2,12)$ & & $1,63(1,34-1,99)$ & \\
\hline 3 ou mais & $2,02(1,65-2,49)$ & & $1,88(1,53-2,31)$ & \\
\hline Problemas de saúde & & $<0,001$ & & $<0,001$ \\
\hline Não & 1,00 & & 1,00 & \\
\hline Sim & $1,82(1,56-2,12)$ & & $1,42(1,21-1,68)$ & \\
\hline Aposentadoria & & $<0,001$ & & 0,01 \\
\hline Não & 1,00 & & 1,00 & \\
\hline Sim & $1,59(1,29-1,96)$ & & $1,40(1,12-1,76)$ & \\
\hline Vínculo com o usuário & & 0,01 & & 0,41 \\
\hline Outros & 1,00 & & 1,00 & \\
\hline Pai/Mãe & $1,41(1,04-1,90)$ & & $1,14(0,85-1,54)$ & \\
\hline Irmão & $1,11(0,78-1,56)$ & & $1,01(0,72-1,42)$ & \\
\hline Cônjuge & $1,31(0,95-180)$ & & $1,11(0,81-1,52)$ & \\
\hline Filho(a) & $1,16(0,83-1,63)$ & & $1,10(0,79-1,52)$ & \\
\hline Único cuidador & & 0,007 & & 0,04 \\
\hline Não & 1,00 & & 1,00 & \\
\hline Sim & $1,22(1,05-1,41)$ & & $1,16(1,00-1,34)$ & \\
\hline Se sente sobrecarregado & & $<0,001$ & & $<0,001$ \\
\hline Não & 1,00 & & 1,00 & \\
\hline Sim & $1,64(1,41-1,90)$ & & $1,49(1,29-1,73)$ & \\
\hline
\end{tabular}

IC95\%: intervalo de 95\% de confiança.

* Conforme modelo hierárquico;

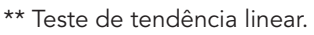

átricos menores pode ser decorrente da ausência de apoio social. Ter alguém com quem dividir o cuidado pode significar uma menor sobrecarga para lidar com eventos e condições produtoras de estresse e, dessa forma, agir como proteção para os transtornos psiquiátricos menores 11. Saber-se amado e cuidado, ter amigos íntimos e confidentes esteve relacionado com baixos níveis de ansiedade, depressão e somatizações 11. Assim melhorando a adaptação para as circunstâncias particulares de estresse e diminuindo o efeito dos eventos produtores desse estresse, mesmo que não sejam evitados, podem passar a ter menores consequências.

A prevalência de transtornos psiquiátricos menores encontrada no presente estudo foi ainda cerca de $50 \%$ maior naqueles familiares que referiram sobrecarga quando comparados aos que não relataram. Embora esse efeito não tenha sido apresentado anteriormente, Tomasi et al. 10 aferiram sobrecarga de cuidadores de usuários de CAPS em Pelotas e encontraram alto grau de correlação com transtornos psiquiátricos menores, também avaliados através do SRQ-20. Assim, os instrumentos utilizados revelaram-se fidedignos para detectar sintomas de transtornos em cuidadores com maior sobrecarga e captar maiores níveis de sobrecarga em indivíduos que apresentaram resultado positivo no SRQ-20.

No entanto, cabe destacar que no presente estudo, com delineamento de corte transversal a exposição e o desfecho estão sendo avaliados no mesmo momento, não sendo possível afirmar que a sobrecarga teria efeito sobre o transtorno psiquiátrico menor ou se aqueles familiares com positividade no SRQ-20 teriam referido sobrecarga com maior probabilidade. Assim, embora a causalidade reversa deva ser considerada como limitação no presente estudo, os resultados da análise ajustada, através de um modelo hierar- 
quizado sugerem que não se devem ao acaso as relações de escolaridade, eventos estressores, problemas de saúde, aposentadoria, único cuidador e sobrecarga autorreferida com transtorno psiquiátrico menor, mesmo que não seja possível conhecer o sentido dessas associações.

Outra limitação a ser considerada refere-se a não ter sido considerada a gravidade do transtorno mental do usuário do CAPS que de alguma forma poderia ter influência na saúde mental do familiar.

Dessa forma, a replicação de estudos com a população de familiares de usuários de CAPS deveria ser conduzida no Brasil, chamando a atenção para a importância da realização de acompanhamentos longitudinais que poderiam ter como linha de base a investigação dos cui-

\section{Resumo}

Com o objetivo de descrever a prevalência de transtornos psiquiátricos menores e sua associação com variáveis sociodemográficas, sobrecarga e eventos estressores autorreferidos, foi conduzido um estudo transversal com 936 familiares cuidadores de usuários de Centros de Atenção Psicossocial. Transtornos psiquiátrico menor foi identificado pelo Self-Reporting Questionnaire (SRQ-20), utilizando para definição de caso o ponto de corte de oito ou mais respostas positivas para mulheres e seis ou mais para homens. Realizaram-se análises bivariada e multivariada por regressão de Poisson. A prevalência de transtornos psiquiátricos menores foi de 49\% (IC95\%: 46\%-52\%). Na análise ajustada, maiores prevalências foram observadas nos familiares com menor escolaridade, com relato de problemas de saúde, com maior número de ocorrência de eventos estressores, aposentados, que são os únicos cuidadores e que referiram sobrecarga. Os resultados indicam uma elevada prevalência de transtornos psiquiátricos menores, identificando algumas associações, o que poderá instrumentalizar serviços de Saúde Mental e Atenção Básica.

Cuidadores; Serviços de Saúde Mental; Saúde Mental dadores a partir da implementação por parte dos serviços de medidas que visem à melhoria no suporte social e a diminuição da sobrecarga. Assim as associações encontradas no presente estudo e outras, como severidade do transtorno apresentado pelo usuário, poderiam estar sendo mais bem avaliadas.

Concluindo, este estudo permitiu a identificação de fatores associados aos transtornos psiquiátricos menores, possibilitando a indicação de medidas de prevenção e controle por meio de políticas públicas propostas nas áreas de Atenção Básica e de Saúde Mental, visando não apenas o portador de transtorno mental ou de outras doenças crônicas, mas todos os usuários dos serviços de saúde sejam eles portadores de alguma patologia ou cuidadores.

\section{Colaboradores}

L. C. M. Quadros e D. P. Gigante contribuíram com a análise e interpretação dos dados, elaboração do rascunho, na revisão crítica do conteúdo e na aprovação da versão final do manuscrito. L. P. Kantorski e V. M. R. Jardim contribuíram com a concepção, planejamento, interpretação dos dados, na elaboração do rascunho, na revisão crítica do conteúdo e participaram da aprovação da versão final do manuscrito. 


\section{Referências}

1. Comissão Organizadora da III CNSM. Relatório final da III Conferência Nacional de Saúde Mental. Brasília: Conselho Nacional de Saúde/Ministério da Saúde; 2002.

2. Ministério da Saúde. Saúde mental no SUS: os Centros de Atenção Psicossocial. Brasília: Ministério da Saúde; 2004.

3. Oliveira RMO, Loyola MC. Família do paciente psiquiátrico: o retrato de uma ilustre desconhecida. Acta Sci Health Sci 2004; 26:213-22.

4. Melman J. Família e doença mental: repensando a relação entre profissionais de saúde e familiares. São Paulo: Escrituras; 2002.

5. Bandeira M, Barroso SM. Sobrecarga das famílias de pacientes psiquiátricos. J Bras Psiquiatr 2005; 54:34-46.

6. Pegoraro RF, Caldana RHL. Sobrecarga de familiares de usuários de um Centro de Atenção Psicossocial. Psicol Estud 2006; 11:569-77.

7. Ludemir AB. Associação dos transtornos mentais comuns com a informalidade das relações de trabalho. J Bras Psiquiatr 2005; 54:198-204.

8. Gonçalves DM, Kapczinski F. Transtornos mentais em comunidade atendida pelo Programa Saúde da Família. Cad Saúde Pública 2008; 24:1641-50.

9. Bruns A, Hilario MO, Jennings F, Silva CA, Natour J. Quality of life and impact of the disease on primary caregivers of juvenile idiopathic arthritis patients. Joint Bone Spine 2008; 2:149-54.

10. Tomasi E, Rodrigues JO, Feijó GP, Facchini LA, Piccini RX, Thumé E, et al. Sobrecarga em familiares de portadores de sofrimento psíquico que freqüentam Centros de Atenção Psicossocial. Saúde Debate 2010; 34:159-67.

11. Costa AG, Ludermir AB. Transtornos mentais comuns e apoio social: estudo em comunidade rural da Zona da Mata de Pernambuco, Brasil. Cad Saúde Pública 2005; 21:73-9.

12. Costa JSD, Menezes AMB, Olinto MTA, Gigante DP, Macedo S, Britto MAP. Prevalência de distúrbios psiquiátricos menores na cidade de Pelotas, RS. Rev Bras Epidemiol 2002; 5:164-73.

13. Ludermir AB, Melo Filho DA. Condições de vida e estrutura ocupacional associadas a transtornos mentais comuns. Rev Saúde Pública 2002; 36: 213-21.
14. Maragno L, Goldbaum M, Gianini RJ, Novaes HMD, Cesar CL. Prevalência de Transtorno mental comum em populações atendidas pelo Programa Saúda da Família (QUALIS) no Município de São Paulo, Brasil. Cad Saúde Pública 2006; 22:1639-48.

15. Harding TW, Arango MV, Baltazar J, Climent CE, Ibrahim HHA, Ladrido-Ignacio L, et al. Mental disorders in primary health care: a study of their frequency and diagnosis in four developing countries. Psychol Med 1980; 10:231-41.

16. Mari JJ, Williams P. A comparison of the validity of two psychiatric screening questionnaires (GHQ12 and SRQ-20) in Brazil, using Relative Operating Characteristic (ROC) analysis. Psychol Med 1985; 15:651-9.

17. Rodrigues-Neto JF, Figueiredo MFS, Faria AAS, Fagundes M. Transtornos mentais comuns e o uso de práticas de medicina complementar e alternativa estudo de base populacional. J Bras Psiquiatr 2008; 57:233-9.

18. Barroso SM, Bandeira MEN. Sobrecarga de familiares de pacientes psiquiátricos atendidos na rede pública. Rev Psiquiatr Clín (São Paulo) 2007; 34:270-7.

19. Fonseca MLG, Guimarães MBL, Vasconcelos EM. Sofrimento difuso e transtornos mentais comuns: uma revisão bibliográfica. Rev APS 2008; 11:285-94.

20. Lopes SC, Faerstein E, Chor D. Eventos de vida produtores de estresse e transtornos mentais comuns: resultados do Estudo Pró-Saúde. Cad Saúde Pública 2003; 19:1713-20.

21. Lima MS, Soares BGO, Mari JJ. Saúde e doença mental em Pelotas, RS: dados de um estudo populacional. Rev Psiquiatr Clin (São Paulo) 1999; 26:225-35.

22. Souza MFM, Silva GR. Risco de distúrbios psiquiátricos menores em área metropolitana na região Sudeste do Brasil. Rev Saúde Pública 1998; 32:50-8.

Recebido em 27/Mai/2011

Versão final reapresentada em 31/Ago/2011

Aprovado em 08/Set/2011 